\title{
LncRNA ARST is a Novel Prognostic and Diagnostic Biomarker for Colorectal Cancer
}

\author{
Lujia Dong ${ }^{1} *$ \\ Di Liu ${ }^{2, *}$ \\ Dongshuai Jing \\ Huihui $X u^{\prime}$ \\ Chenxiao Zhang' \\ Donglei Qi' \\ Dechun Liu' \\ 'Department of Gastrointestinal Surgery, \\ The First Affiliated Hospital, and College \\ of Clinical Medicine of Henan University \\ of Science and Technology, Luoyang City, \\ Henan Province, 47I003, People's \\ Republic of China; ${ }^{2}$ Department of \\ Thyroid Surgery, The First Affiliated \\ Hospital, and College of Clinical Medicine \\ of Henan University of Science and \\ Technology, Luoyang City, Henan \\ Province, 47I003, People's Republic of \\ China \\ *These authors contributed equally to \\ this work
}

Correspondence: Dechun Liu Department of Gastrointestinal Surgery, The First Affiliated Hospital, and College of Clinical Medicine of Henan University of Science and Technology, No. 24 Jinghua Road, Jianxi District, Luoyang City, Henan Province, 47I003, People's Republic of China

Email liudechunluoyang@I63.com
Background: ALDOA-related specific transcript (ARST) is a recently identified long noncoding RNA (lncRNA) that suppresses glioma progression, while its role in other cancers is unclear. This study explored the role of ARST in colorectal cancer (CRC).

Methods: The present study included $60 \mathrm{CRC}$ patients, 60 patients with colon polyps (CP), 60 colitis patients, 60 hemorrhoid patients and 60 healthy controls. All participants were subjected to the collection of plasma, and paired CRC and non-tumor tissues were collected from CRC patients. All samples were subjected to RNA isolation and RT-qPCR to detect the expression of ARST. ROC curve and survival curve analysis were performed to evaluate the diagnostic and prognostic values of plasma ARST for CRC.

Results: The expression levels of ARST were lower in CRC plasma samples compared to that in the patient groups and controls $(p<0.01)$, while other patient groups and controls showed no significant difference. The expression levels of ARST were also lower in CRC tissues compared to that in non-tumor tissues $(p<0.01)$. Plasma expression levels of ARST effectively distinguished CRC patients from other patients and controls. The expression levels of ARST were closely correlated with patients' survival. Chi-squared test analysis showed that ARST was closely associated with patients' distant metastasis but not tumor size.

Conclusion: ARST is downregulated in $\mathrm{CRC}$, and it might be applied in the diagnosis and prognosis of CRC.

Keywords: ALDOA-related specific transcript, colorectal cancer, diagnosis, prognosis

\section{Introduction}

Colorectal cancer (CRC), also known as colon cancer and bowel cancer, is the 3rd most common type of malignancy in both males and females. ${ }^{1,2}$ It is estimated that CRC affects about 1 out of 25 females and 1 out of 23 males during their lifetime. ${ }^{3}$ With proper treatment and diagnosis at early stages, around $90 \%$ of CRC patients could survive more than 5 years. ${ }^{4,5}$ However, early diagnosis of CRC is difficult, and most of CRC patients $(60 \%)$ are diagnosed at advanced stages, resulting in poor prognosis. ${ }^{6}$ In many cases, CRC can be misdiagnosed as other clinical disorders in colon, such as colon polyps (CP), colitis and hemorrhoid. ${ }^{7,8}$

Although CRC and other colon diseases have similar symptoms, different molecular pathways are involved. ${ }^{7,8}$ Therefore, identification of differentially expressed molecular factors may facilitate the development of novel diagnostic biomarkers for CRC. ${ }^{8,9}$ For instance, miRNAs and carcinoembryonic antigen (CEA) have been developed to detect CRC at early stages. ${ }^{10,11}$ However, these biomarkers are limited by the low sensitivity and specificity. Long non-coding 
RNAs (lncRNAs) do not encode proteins but regulate the transcription or/and translation of genes to participate in cancer development and progression. ${ }^{12,13}$ In cancer biology, certain lncRNAs play critical roles and can be used as potential targets for the treatment and diagnosis of cancers. $^{12,13}$ ALDOA-related specific transcript (ARST) is a recently identified lncRNA that is downregulated in glioma. ${ }^{14}$ Moreover, ALDOA inhibits glioma progression by regulating actin cytoskeleton integrity. However, its role in other cancers is unclear. This study explored the role of ARST in CRC.

\section{Materials and Methods}

\section{Patients and Healthy Controls}

From May 2014 to August 2016, 60 CRC patients (22 females and 38 males, $54.6 \pm 6.7$ years old), 60 patients with colon polyps (CP, 22 females and 38 males, $54.8 \pm$ 6.4 years old), 60 colitis patients (22 females and 38 males, $54.6 \pm 6.9$ years old), 60 hemorrhoid patients (22 females and 38 males, $54.4 \pm 6.6$ years old) and 60 healthy controls (22 females and 38 males, $54.9 \pm 6.9$ years old) were enrolled at the First Affiliated Hospital, and College of Clinical Medicine of Henan University of Science and Technology. This study was approved by the Ethics Committee of this hospital. All experimental procedures were carried out in compliance with the Declaration of Helsinki and Good Clinical Practices. CRC patients were diagnosed through colonoscopy, through which biopsy was performed. CRC tumor tissues were confirmed by histopathological analysis. CP was also diagnosed by colonoscopy. Colitis was diagnosed by Sigmoidoscopy and the analysis of bowel inflammation. Hemorrhoid was diagnosed by checking the area around anus, and a digital rectal exam was also performed in some cases. Malignant tumors were excluded from CP, colitis and hemorrhoid patients by performing histopathological analysis. Healthy controls were enrolled after they completed systemic physiological examinations, and no abnormalities were observed. All participants signed the informed consent.

\section{A 5-Year Follow-Up}

The survival of $60 \mathrm{CRC}$ patients was monitored after admission. All patients were followed up every month through telephone or outpatient visit. Survival data were summarized to perform survival analysis.

\section{Collection of Samples}

To analyze differential expression of ARST, CRC and paired tissues were collected from all $60 \mathrm{CRC}$ patients. Tissue samples were collected by dissecting the resected tumors of biopsies. Tissue samples were confirmed by histopathological exam. On the day of admission, blood was collected from all patients and healthy controls under fasting conditions. Blood samples were centrifuged in EDTA tubes, and the supernatant (plasma) was collected. After collection, all samples were stored in liquid nitrogen before the subsequent assays.

\section{RNA Preparations}

Total RNAs were isolated using MagNA Pure Compact RNA Isolation Kit (Roche Life Science). In brief, 1 volume of sample was subjected to lysis using 10 volume lysis buffer in a cold room for $20 \mathrm{~min}$. After centrifugation at $1200 \mathrm{~g}$ for $10 \mathrm{~min}$, the supernatant was transferred to binding column, followed by centrifugation at $12,000 \mathrm{~g}$ for 1 min to achieve RNA binding. After washing with washing buffer, RNA elution was performed using RNase-free water. RNA samples were stored at $-80{ }^{\circ} \mathrm{C}$ before use.

\section{RT-qPCR for Gene Expression Analysis}

RNA samples were subjected to DNase I digestion to remove gDNA, followed by the analysis of integrity and quantification using Bioanalyzer (2100). About 2000 ng RNA samples were used to prepare cDNA samples using SSRT IV system (Invitrogen). Gene expression levels were measured by qPCRs with $18 \mathrm{~S}$ rRNA as the internal control. $\mathrm{Ct}$ values were normalized to $18 \mathrm{~S}$ rRNA using the $2^{-\Delta \Delta \mathrm{CT}}$ method.

\section{Statistical Analysis}

The GraphPad Prism 7.0 (GraphPad) software was used to analyze data and prepare images. All experiments were performed in triplicate manner. Wilcoxon signed-rank test, Mann-Whitney $U$-test or two-tailed Student's $t$-test were used to explore statistical significance. The potential value of ARST in the diagnosis of CRC was explored by performing ROC curve analysis, in which CRC patients were included as true positive cases, and the true negative cases were $\mathrm{CP}$ patients, colitis patients, hemorrhoid patients or healthy controls. The $60 \mathrm{CRC}$ patients were grouped into high and low ARST level groups, with 30 patients in each group. Associations between plasma expression levels of ARST and patients' clinical data were analyzed by Chi-squared $t$-test. 
Using the 5-year follow-up data, survival curves were plotted for both groups and compared by Log rank test to explore the prognostic value of ARST for CRC. $p<0.05$ was statistically significant.

\section{Results}

\section{Differential Expression of ARST in CRC}

Plasma samples derived from $60 \mathrm{CRC}$ patients, 60 patients with colon polyps (CP), 60 colitis patients, 60 hemorrhoid patients and 60 controls were subjected RT-qPCRs to detect the differential expression of ARST. It showed that the expression levels of ARST were lower in CRC plasma compared to that in the patient groups and controls, while other patient groups and controls showed no significant difference (Figure 1A, $p<0.01$ ). CRC and paired non-tumor tissue samples were also subjected to RTqPCRs, and the results showed that the expression levels of ARST were also lower in CRC tissues compared to that in non-tumor tissues (Figure 1B, $p<0.01$ ).

\section{Diagnostic Value of ARST for CRC}

The potential value of ARST in the diagnosis of CRC was explored by performing ROC curve analysis, in which CRC patients were included as true positive cases and the true negative cases were $\mathrm{CP}$ patients (Figure 2A), colitis patients (Figure 2B), hemorrhoid patients (Figure 2C) or healthy controls (Figure 2D), respectively. Area under the curve (AUC) higher than 0.65 indicated potential diagnostic values. In all 4 cases, AUC was higher than 0.65. Therefore, ARST effectively distinguished CRC patients from other patients and controls.

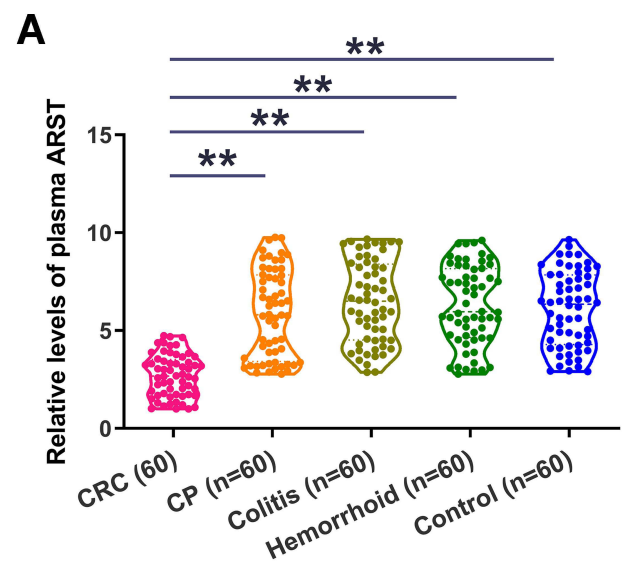

\section{Potential Value of ARST in the Prediction of Patients' Death}

The 60 CRC patients were grouped into high and low ARST level groups, with 30 patients in each group. Using the 5-year follow-up data, survival curves were plotted for both groups and compared by Log rank test to explore the prognostic value of ARST for CRC (Figure 3). Compared to patients in the low expression level group, patients with high expression levels of ARST experienced significantly increased death rate and reduced survival time during the 5-year follow-up, suggesting that reduced expression levels of ARST in plasma were closely correlated with patients' poor survival.

\section{Associations Between Plasma Expression Levels of ARST and Patients' Clinical Data}

The 60 CRC patients were grouped into high and low ARST level groups, with 30 patients in each group. Associations between plasma expression levels of ARST and patients' clinical data were analyzed by Chi-squared $t$-test. Chi-squared test results showed that ARST was closely associated with patients' distant metastasis but not tumor size (Table 1).

\section{Discussion}

This study explored the involvement of IncRNA ARST in CRC. We found that the expression of ARST is altered in CRC, and it may serve as a potential diagnostic and prognostic biomarker for CRC. In addition, ARST may regulate the metastasis of CRC to affect CRC progression.

B

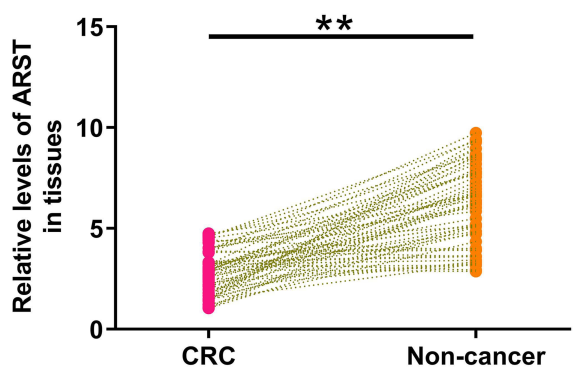

Figure I Differential expression of ARST in CRC. Plasma samples derived from 60 CRC patients, 60 patients with colon polyps (CP), 60 colitis patients, 60 hemorrhoid patients and 60 controls (A) as well as paired CRC and non-tumor tissue samples from CRC patients (B) were subjected to the preparation of RNA samples. RT-qPCRs were performed using all RNA samples to analyze the differential expression of ARST. $* * p<0.01$. 

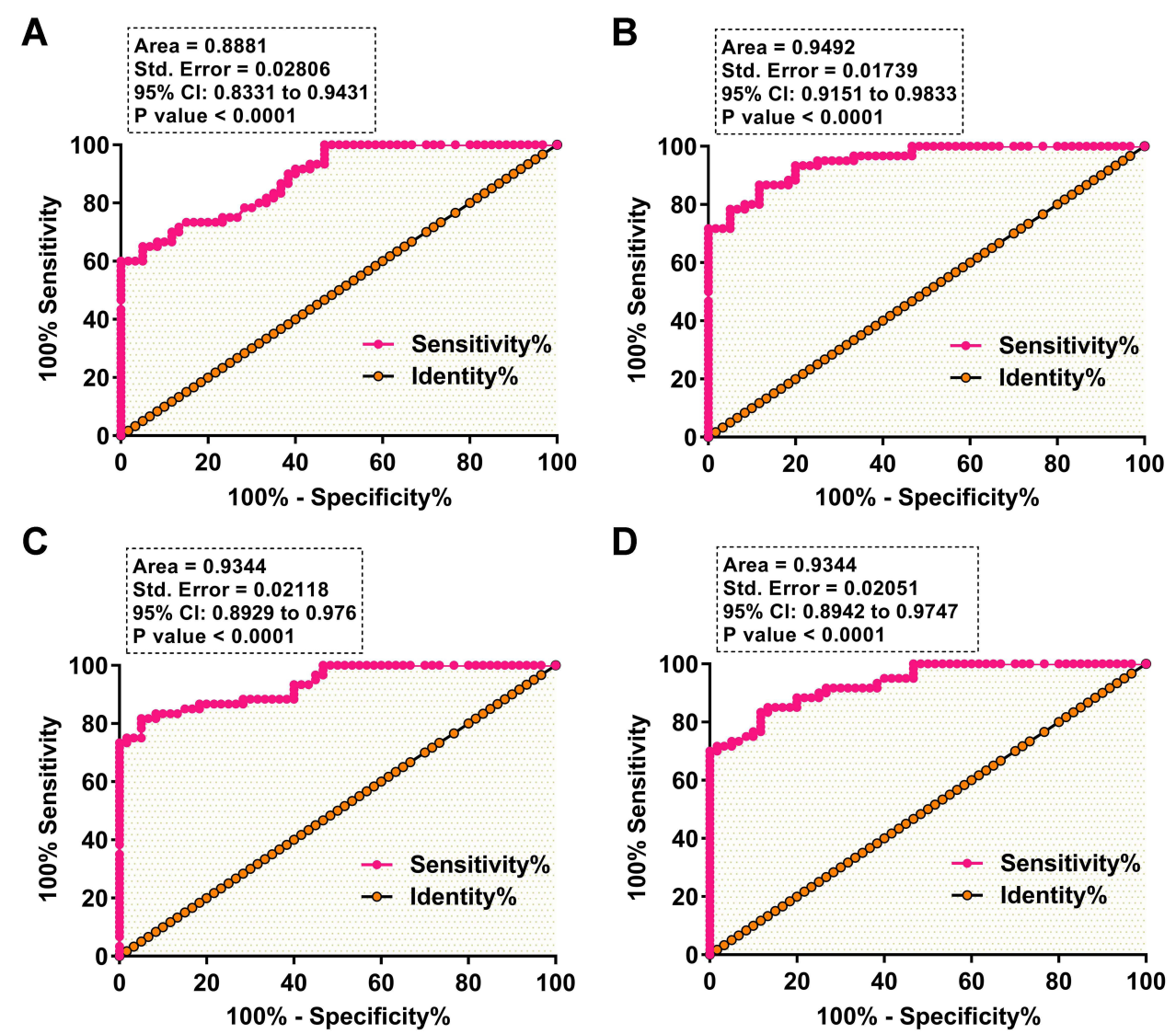

Figure 2 Diagnostic value of plasma ARST for CRC. The potential value of plasma ARST in the diagnosis of CRC was explored by performing ROC curve analysis, in which CRC patients were included as true positive cases and the true negative cases were CP patients (A), colitis patients (B), hemorrhoid patients (C) or healthy controls (D). Area under the curve (AUC) was presented.

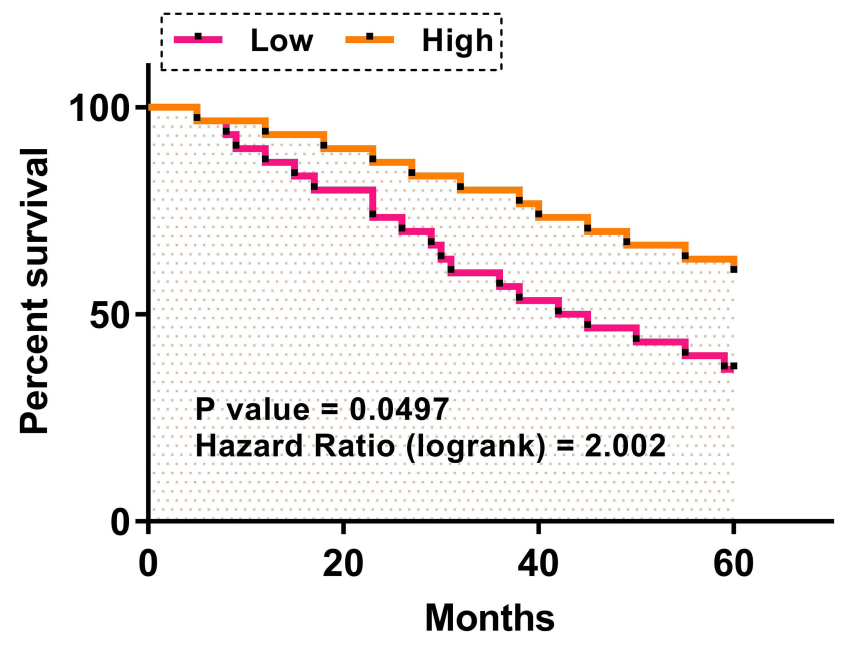

Figure 3 Potential value of plasma ARST in the prediction of patients' death. The 60 CRC patients were grouped into high and low plasma ARST level groups, in each group 30 patients were included. Using the 5-year follow-up data, survival curves were plotted for both groups and compared by Log rank test to explore the prognostic value of plasma ARST for CRC.

ARST is a recently identified lncRNA in glioma. ${ }^{14}$ It is reported that the expression levels of ARST were low in glioma and overexpression of ARST suppressed the actin cytoskeleton integrity mediated by ALDOA to suppress glioma progression. ${ }^{14}$ Based on our knowledge, the involvement of ARST in other types of cancer and human disease is unclear. This study revealed downregulation of ARST in CRC but not in other clinical disorders of colon. Therefore, ARST is likely a tumor suppressor in CRC. Although we did not explore the role of ARST in regulating the behaviors of CRC cells, our chi-squared test results showed that ARST was only closely correlated with distant tumor metastasis but not tumor size. Therefore, ARST is likely involved in tumor metastasis but not tumor growth of CRC. However, future studies are needed to explore the functions of ARST in CRC and analyze possible mechanisms that mediate its function.

$\mathrm{CRC}$ at early stages may mimic the symptoms of $\mathrm{CP}$, colitis and hemorrhoid. These symptoms may include lumps and rectal bleeding in the anus. ${ }^{15}$ Therefore, misdiagnosis that leads to delayed treatment and cancer progression is common. In this study, we showed that the expression levels of ARST were low in CRC but not 
Table I Associations Between Plasma ARST Levels and Patients' Clinical Data

\begin{tabular}{|c|c|c|c|c|c|}
\hline \multirow[t]{2}{*}{ Characteristics } & \multirow[t]{2}{*}{ Number } & \multicolumn{2}{|c|}{ ARST Expression } & \multirow[t]{2}{*}{ Chi-Squared } & \multirow[t]{2}{*}{$P$ value } \\
\hline & & Low $(n=30)$ & High $(n=30)$ & & \\
\hline \multicolumn{6}{|l|}{ Age (years) } \\
\hline$\geq 55$ & 29 & 13 & 16 & 0.6 & 0.44 \\
\hline$<55$ & 31 & 17 & 14 & & \\
\hline \multicolumn{6}{|l|}{ Gender } \\
\hline Male & 38 & 18 & 20 & 0.29 & 0.59 \\
\hline Female & 22 & 12 & 10 & & \\
\hline \multicolumn{6}{|l|}{ Depth of tumor } \\
\hline $\mathrm{T} 3+\mathrm{T} 4$ & 34 & 16 & 18 & 0.27 & 0.6 \\
\hline $\mathrm{TI}+\mathrm{T} 2$ & 26 & 14 & 12 & & \\
\hline \multicolumn{6}{|l|}{ Distant metastasis } \\
\hline Yes & 15 & 12 & 3 & 7.2 & 0.007 \\
\hline No & 45 & 18 & 27 & & \\
\hline \multicolumn{6}{|l|}{ Tumor size $(\mathrm{cm})$} \\
\hline$>5$ & 34 & 20 & 14 & 2.44 & 0.12 \\
\hline$\leq 5$ & 26 & 10 & 16 & & \\
\hline \multicolumn{6}{|c|}{ Tumor differentiation } \\
\hline Well & 12 & 5 & 7 & 0.67 & 0.71 \\
\hline Moderate & 33 & 18 & 15 & & \\
\hline Poor & 15 & 7 & 8 & & \\
\hline
\end{tabular}

in $\mathrm{CP}$, colitis and hemorrhoid. In addition, ROC curve analysis showed that altered plasma expression of ARST effectively separated CRC patients from CP patients, colitis patients and hemorrhoid patients. In addition, low plasma expression levels of ARST were closely correlated with patients' poor survival. Therefore, analyzing the expression pattern of ARST prior to the diagnosis of CRC may avoid misdiagnosis and identify patients with high risk of death to prolong their survival. However, this study only included $60 \mathrm{CRC}$ patients, and the sample size is small. Our conclusions remain to be verified by future studies with bigger sample sizes.

The present study only explored the clinical value of ARST in the diagnosis and prognosis of CRC. However, the role of ARST in regulating CRC cell behaviors and the mechanisms is unknown. More studies are needed to cover this topic.

In conclusion, ARST is downregulated in CRC, and it may serve as a potential diagnostic and prognostic biomarker for CRC.

\section{Data Sharing Statement}

The data that support the findings of this study are available on request from the corresponding author. The data are not publicly available due to their containing information that could compromise the privacy of research participants.

\section{Ethical Approval and Consent to Participate}

All patients signed the written informed consent. All procedures were approved by the First Affiliated Hospital, and College of Clinical Medicine of Henan University of Science and Technology Ethics Committee. Procedures operated in this research were completed in keeping with the standards set out in the Announcement of Helsinki and laboratory guidelines of research in China.

\section{Funding}

There is no funding to report.

\section{Disclosure}

The authors declare that they do not have any commercial or associative interest that represents a conflict of interest in connection with the work submitted.

\section{References}

1. Siegel RL, Miller KD, Goding Sauer A, et al. Colorectal cancer statistics, 2020. CA Cancer J Clin. 2020;70(3):145-164. doi:10.3322/ caac. 21601 
2. Siegel RL, Miller KD, Fedewa SA, et al. Colorectal cancer statistics, 2017. CA Cancer J Clin. 2017;67(3):177-193. doi:10.3322/ caac. 21395

3. Granados-Romero J, Valderrama-Treviño A, Contreras Flores E, et al. Colorectal cancer: a review. Int J Res Med Sci. 2017;5:4667. doi:10.18203/2320-6012.ijrms20174914

4. Rejhová A, Opattová A, Čumová A, Slíva D, Vodička P. Natural compounds and combination therapy in colorectal cancer treatment. Eur J Med Chem. 2018;144:582-594. doi:10.1016/j.ejmech.2017.12.039

5. Modest DP, Pant S, Sartore-Bianchi A. Treatment sequencing in metastatic colorectal cancer. Eur $J$ Cancer. 2019;109:70-83. doi:10.1016/j.ejca.2018.12.019

6. Loree JM, Kopetz S. Recent developments in the treatment of metastatic colorectal cancer. Ther Adv Med Oncol. 2017;9(8):551-564. doi:10.1177/1758834017714997

7. Kotoula V, Fostira F, Fountzilas E. Primary resistance to immune checkpoint inhibitors in metastatic colorectal cancer-beyond the misdiagnosis. JAMA Oncol. 2019;5(5):740-741. doi:10.1001/ jamaoncol.2019.0521

8. Rad AH, Aghebati-Maleki L, Kafil HS, Abbasi A. Molecular mechanisms of postbiotics in colorectal cancer prevention and treatment. Crit Rev Food Sci Nutr. 2021;61(11):1787-1803.

9. Al-Sohaily S, Biankin A, Leong R, Kohonen-Corish M, Warusavitarne J. Molecular pathways in colorectal cancer. $J$ Gastroenterol Hepatol. 2012;27(9):1423-1431. doi:10.1111/j.1440-1746.2012.07200.x
10. Huang Z, Huang D, Ni S, Peng Z, Sheng W, Du X. Plasma microRNAs are promising novel biomarkers for early detection of colorectal cancer. Int J Cancer. 2010;127(1):118-126. doi:10.1002/ ijc. 25007

11. Lech G, Słotwiński R, Słodkowski M, Krasnodębski IW. Colorectal cancer tumour markers and biomarkers: recent therapeutic advances. World J Gastroenterol. 2016;22(5):1745-1755. doi:10.3748/wjg.v22. i5.1745

12. Zhang Y, Tang L. The application of IncRNAs in cancer treatment and diagnosis. Recent Pat Anticancer Drug Discov. 2018;13 (3):292-301. doi:10.2174/1574892813666180226121819

13. Wu P, Mo Y, Peng M, et al. Emerging role of tumor-related functional peptides encoded by lncRNA and circRNA. Mol Cancer. 2020;19 (1):22. doi:10.1186/s12943-020-1147-3

14. Sun J, He D, Fu Y, et al. A novel lncRNA ARST represses glioma progression by inhibiting ALDOA-mediated actin cytoskeleton integrity. J Exp Clin Cancer Res. 2021;40(1):187. doi:10.1186/ s13046-021-01977-9

15. Mounsey AL, Halladay J, Sadiq TS. Hemorrhoids. Am Fam Physician. 2011;84(2):204-210.

\section{Publish your work in this journal}

Cancer Management and Research is an international, peer-reviewed open access journal focusing on cancer research and the optimal use of preventative and integrated treatment interventions to achieve improved outcomes, enhanced survival and quality of life for the cancer patient.
The manuscript management system is completely online and includes a very quick and fair peer-review system, which is all easy to use. Visit http://www.dovepress.com/testimonials.php to read real quotes from published authors. 